\title{
Combat triage support using the Internet of Military Things
}

\author{
Michal Dyk \\ Faculty of Cybernetics \\ Military University of Technology \\ Warsaw, Poland \\ michal.dyk@wat.edu.pl
}

\author{
Mariusz Chmielewski \\ Faculty of Cybernetics \\ Military University of Technology \\ Warsaw, Poland \\ mariusz.chmielewski@wat.edu.pl
}

\author{
Andrzej Najgebauer \\ Faculty of Cybernetics \\ Military University of Technology \\ Warsaw, Poland \\ andrzej.najgebauer@wat.edu.pl
}

\begin{abstract}
Triage on the battlefield is a very challenging task. Life of the wounded soldiers depends on the efficiency of this process and there is still lack of supporting solutions. This paper presents a new approach for using Internet of Military Things in combat triage. We propose an ontological approach to evaluate soldiers' health state and information framework which allows first responders and commanders to query the sensor network for needed information. Some simulation experiments were conducted, which results show that the proposed method can be applied in highly distributed and heterogeneous environment of the smart devices on the battlefield.
\end{abstract}

\section{INTRODUCTION}

$\mathbf{T}$ RIAGE of casualties is important part of the modern military operations. It is a proven method of providing medical care in situations where available resources are not sufficient. It is also a process which gives commanders information about troops ability to accomplish the mission. In this paper we propose a method for supporting combat triage process using devices connected into Internet of Military Things. Its goal is to monitor and provide information about soldiers health status for commanders and medical support like field medics. An important feature of this method is that it is information-centric, so user can define information need and the role of the smart devices is to fulfil this need. All processing of the raw data is done in distributed environment of the sensors network. Using this method triage process can be conducted with different types of sensors which monitor different vital signs of soldiers, and user does not need to be an medical expert to analyse readings.

\section{Combat Triage}

In a healthcare triage is a process of categorizing criticality of patient's condition [1]. The person responsible for triage performs a brief, focused assessment and assigns the patient a triage acuity level, which is a proxy measure of how long an individual patient can safely wait for a medical screening examination and treatment [2]. Such process is conducted especially when the demand for medical care overwhelms the available resources. In such cases first responders perform triage of casualties to ensure that they receive treatment in ordered way, depending on their health status.
Civilian and combat triage has a lot in common. Both of them refer to crisis situations and use similar methods. For instance, in the USA, The National Association of Emergency Medical Technician's (NAEMT) adopts a military Tactical Combat Casualty Care (TCCC) course to train civilian Emergency Medical Services (EMS) [3]. However there are some factors which make differences between civilian and combat triage. According to TCCC [4], those are:

- Hostile fire,

- Darkness,

- Environmental extremes,

- Different wounding epidemiology,

- Limited equipment,

- Need for tactical maneuver,

- Long delays to hospital care,

- Different medic training and experience.

All those factors cause that the combat triage, in many cases, is more challenging than civilian one. Especially first responders, medics and patients themselves are under constant threat. It is also worth pointing out that during combat operations, the patient is only part of the mission, where in civilian setting patient is the mission. That is why the combat triage has, in fact, three goals [4]:

1) Treat the casualty.

2) Prevent further casualties.

3) Complete the mission.

NATO's AJP-4.10(A) standard [5] defines situation in which triage should be conducted as a Mass Casualty (MASCAL) situation in which an excessive disparity exists between the casualty load and the medical capacities locally available for its management. In such situation principle of treatment may, mainly at the onset of the medical response, change from one based on the individual needs of each patient to one based on the greatest good for the greatest number. That is why NATO standard defines following triage priorities:

1) Immediate Treatment (Group T1). To consist of those requiring emergency care and life-saving surgery. These procedures should not be time-consuming and should concern only those patients with high chances of survival.

2) Delayed Treatment (Group T2). To consist of those in 
need of surgery, but whose general condition permits delay in surgical treatment without unduly endangering life.

3) Minimal Treatment (Group T3). To consist of those with relatively minor injuries who can effectively care for themselves or who can be helped by untrained personnel.

4) Expectant Treatment (Group T4). This group comprises of patients who have received serious and often multiple injuries, and whose treatment would be time-consuming and complicated, with a low chance of survival. If fully treated they make heavy demands on medical manpower and supplies. Until the MASCAL situation is under control, they will receive appropriate supportive treatment. The extent of treatment will depend on available supplies and manpower and may involve the use of large doses of narcotic analgesics. For these patients every effort should be devoted to their comfort, and the possibility of survival with even alarming injuries.

Triage and especially its combat version is a very challenging task, where proper diagnosis and classification of patients are crucial. All decisions must be taken within a very short time and with maximum certainty. Triage is also a continuous process, which means that even when all casualties are prioritized, they need to be monitored constantly, because they state may change. All these challenges cause that there is a great need for triage support.

\section{A. Information framework}

In the presented method a user, which might be commander or field medic or any other person involved in triage process, can define information need which should be fulfilled by the smart sensors. Depending on the situation on the battlefield, number of commanded soldiers, combat intensity and mission goals such need might be different. That is why we propose flexible approach in which responses of the system are not predefined, but rather network of devices works as a kind of information search engine, however restricted to the triage domain.

To describe an information need we use the infon theory proposed by Keith Devlin [6]. Infons are items of information. In its basic form it can be understood as a fact that some objects $a_{1}, \ldots, a_{n}$ are in some relation $R$. It is formally defined as follows:

$$
\sigma=\ll R, a_{1}, \ldots, a_{n}, i \gg
$$

where $R$ is an n-place relation and $a_{1}, \ldots, a_{n}$ are objects appropriate for $R$. Element $i$ is called infon polarity and takes value 1 if objects $a_{1}, \ldots, a_{n}$ are in fact in relation $R$ and 0 otherwise. Infon description can be extended by adding elements which describe spatial $l$ and temporal $t$ location:

$$
\sigma=\ll R, a_{1}, \ldots, a_{n}, l, t, i \gg .
$$

Having that it is possible to indicate that given objects are in relation $R$ at location $l$ and/or time $t$.

Infons are atomic items of information which are used to build more complex sentences called situations [7]. According to the Devlin's theory, situations are natural source of information about the world. Only in particular situation one can state that given infon is factual. To denote that some infon $\sigma$ is an item of information that is true of situation sit the following notion is used:

$$
\text { sit } \models \sigma
$$

It should be read as "sit supports $\sigma$ ". Situation sit in this case is not a part of the real world. We call it an abstract situation which is a mathematical construct. Of course there is an intuitive sense in which to every real situation corresponds an abstract one. Abstract situation in such context is a set of infons:

$$
\{\sigma \mid \text { sit } \models \sigma\}
$$

The construct of abstract situation gives a framework to describe situations in a formal manner on a desired level of complexity. For every real situation it possible to define more ore less sophisticated description using infons. Situations may be "static", which means that they involve one spatial and temporal location (or a number of contemporary spatial locations) or they may be "dynamic" which means that they are spread over a time sequence of locations.

As long as abstract situation is just a mathematical construct, some restrictions should be imposed. The most important is the coherence. An abstract situation sit is said to be coherent if it satisfies the following three conditions:

1) for no $R, a_{1}, \ldots, a_{n}$ is the case that:

$$
\begin{aligned}
& \text { sit } \models \ll R, a_{1}, \ldots, a_{n}, 1 \gg \\
& \text { sit } \models \ll R, a_{1}, \ldots, a_{n}, 0 \gg ;
\end{aligned}
$$

2) if for some $a, b$ it is the case that:

$$
\text { sit } \models \ll \text { same, } a, b, 1 \gg
$$

then $a=b$;

3) for no $a$ is it a case that:

$$
\text { sit } \models \ll \text { same, } a, a, 0 \gg .
$$

From this point we can say that the need for information can be formally described by an abstract situation using presented notation of infons. To make such description as flexible as possible it is important to introduce parameters into infons. Each infon can be parametrized using one of the basic types:

- $T I M:$ the type of temporal location;

- $L O C$ : the type of a spatial location;

- $I N D:$ the type of an individual;

- REL : the type of an relation;

- $P O L$ : the type of polarity (i. e. the 'truth values' 0 and $1)$.

Those types of parameters correspond to the cognitive abilities of the smart devices which allow them to individualize uniformities of the world at the basic level. For each object $x$ there is at least one type such that $x$ is of that type. For example, if $t$ is temporal location, then $t$ is of type TIM. Having that it is possible to construct more generic infons like:

$$
\sigma=\ll \text { atPosition, } \dot{p}, l, 1 \gg
$$


which 'says' that some individual $\dot{p}: I N D$ is at some position $l$. Naturally there is need for some mechanism, which assigns values for parameters. From mathematical point of view such role play anchors. Formally an anchor for set $A$ of parameters is a function defined on $A$ which assigns to each parameter $T_{n}$ in $A$ an object of type $T$. For previously presented infon we can write:

$$
\sigma=\ll \text { atPosition, } f(\dot{p}), l, 1 \gg
$$

where $f(\dot{p})$ return an individual which, in fact, is at position $l$. In presented method the devices connected into IoT act like anchors, so their responsibility is to populate parametric infons with data relevant to the situation which they observe. In other words, they need to answer whether given abstract situation is actual or not. Abstract situation sit is considered actual if:

- sit is coherent situation,

- whenever sit $\models \ll R, a_{1}, \ldots, a_{n}, 1 \gg$ then in the real world it is really the case that $a_{1}, \ldots, a_{n}$ stand in relation $R$,

- whenever sit $\models \ll R, a_{1}, \ldots, a_{n}, 0 \gg$ then $a_{1}, \ldots, a_{n}$ really do not stand in the relation $R$.

By using presented framework it is possible to build different information needs. Some examples, in terms of triage, are shown below:

1) Is Soldierl in condition immediate?

- Information need: sit ${ }^{\prime} \models \ll$ immediate, Soldier $1, \dot{x} \gg$

- Response: sit $\models \ll$ immediate, Soldier $1,1 \gg$

2) What is the state of Soldier1?

- Information need: sit $^{\prime} \models \ll \dot{r}$, Soldier $1,1 \gg$

- Response: sit $\models \ll$ deleyed, Soldier $1,1 \gg$

3) Which soldiers are in state minimal treatement?

- Information need: sit $\models \ll$ minimal, $h, 1 \gg$

- Response: sit $\models \ll$ minimal, Soldier $1,1 \gg$ $\wedge \ll$ minimal, Soldier $2,1 \gg$ $\wedge \ll$ minimal, Soldier $5,1 \gg$

Important in this approach is that, the user does not need to know what kind of sensors are used for soldiers health state monitoring. Devices are responsible for analysing the need, identifying which object/properties are important and answering the question. It means that devices must have some cognitive capabilities and understand (at least in terms of given domain) what they observe. We propose an ontology as a method for handling device's knowledge.

\section{B. IoT triage ontology}

In order to perform classification with the system we have designed a problem solving ontology which represents some of the domain concepts of sensor and medical domains. The

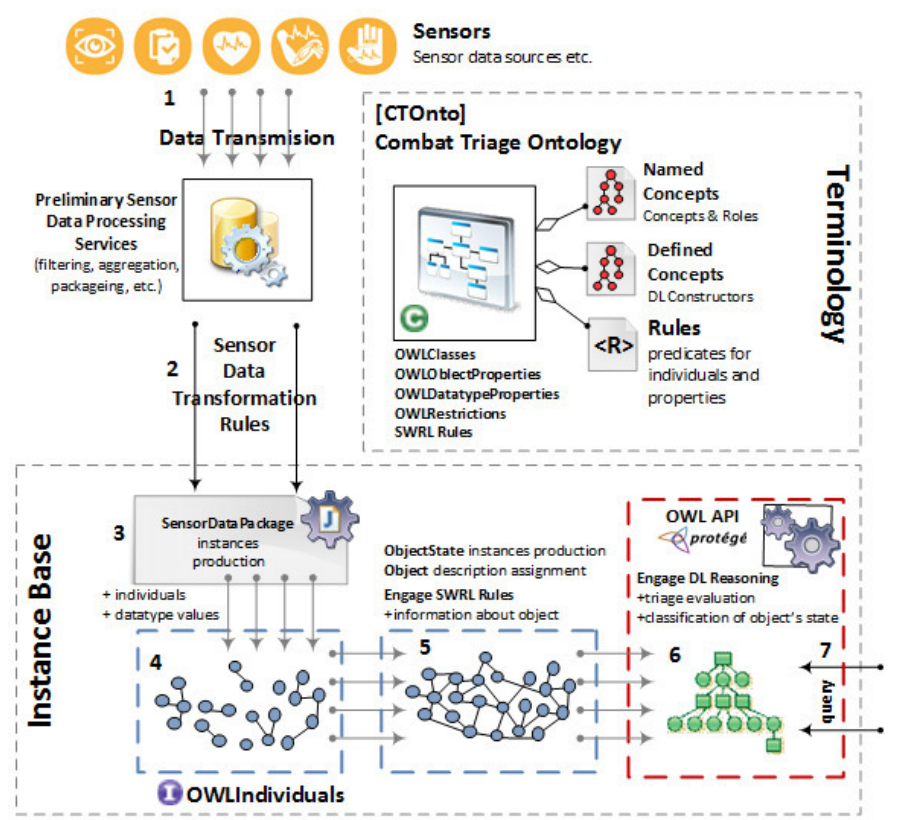

Fig. 1. Sensor Data processing path deliver seven stage combat triage evaluation proces classyfying given batlespace object

utilization of ontology model forms terminology and model constraints on which a triage knowledge base is formulated. In presented system, the knowledge base utilizes Description Logic and First Order logic [8] reasoning techniques delivered by the Pellet reasoner [9]. Knowledge base supported by the reasoning mechanisms deliver model consistency check of instance base (data) ensuring valid relations between data instances, performing classification tasks for instance data and executing rules to infer new facts in the knowledge base. These tasks have been used as tools solving the problem of classifying health state of an individual based on the sensor data measuring stimuli. Implemented in otology concept definitions as well as rules perform data classification tasks evaluating sensor data introduced within the system. The evaluation process takes preliminary data package and confronts the data with "evaluation rules" which analyse specific characteristics of data (discrete or continuous in nature), in order to aggregate and produce information about the inspected (monitored) object. In order to perform the analysis rules contain evaluation or decision predicates. The predicates determine if calculated characteristic contains useful information in context of evaluated object and the environment, e.g. photopletysmography sensor data containing photopletysmogram can be processed to evaluate heart rate, which depending on the object's age, physiological stamina can determine, the stress level, exhaustion and health state. The ontology in that matter offers a set of rules which interpret sensor data (inertial, biomedical) in order to evaluate object's characteristics in context of current health state. The Combat Triage Ontology delivers means to produce more than one predicate based on one instance of SensorDataPackage, which can help to evaluate sensor 


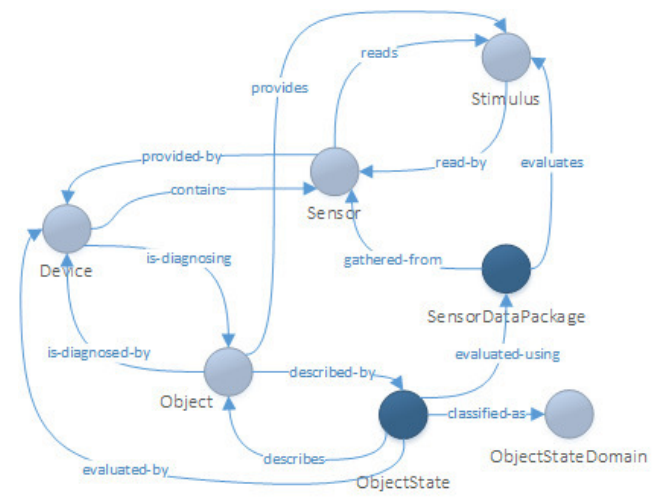

Fig. 2. Main concepts and relations in Combat Triage Ontology formulating basic terminology for diagnosing Object state based on the sensor data analysis

data in context of a health state but also other monitored object's characteristics. The next stage of processing is aimed at evaluating the ObjectState based on the series of associated SensorDataPackages. ObjectState concept holds several DL constructors, which assign to each and every ObjectState the ObjectStateDomain value. The ObjectStateDomain is a nominal concept (enumeration) which holds evaluation statuses for any monitored object. The evaluation status can be understood as an outcome of evaluation process produced by the reasoning mechanism. The classification mechanism in the knowledge base is iteratively performing sensor data analysis after which instances of ObjectState are evaluated and linked with adequate ObjectStateDomain value. The ObjectStateDomain concept provides detailed taxonomy leading towards HumanStateDomain, TriageHumanStateDomain and further specialized according to AJP-4.10.A standard (AJP4.10A-TriageHumanStateDomain), S.M.A.R.T. (S.M.A.R.TTriageHumanStateDomain). Depending on the Object type and aim of object's state evaluation the reasoner is able to evaluate particular ObjectState with status taken from the AJP-4.10A combat triage standard. Having such result the reasoner is able to evaluate instances of SensorDataPackages, engage a set of rules which produce information about given monitored Object, confront sensor information with the context in which the Object is found with respect to the environment (e.g. combat mission, medical treatment, etc.).

The knowledge base utilizes also built-in semantic mapping between enumerated values. This feature is useful for mapping purposes, in which well-defined concept or an instance has a corresponding entity - synonym or entity of very similar semantics. In case of Combat Triage Ontology semantic mapping has been used to map various triage standards and approaches between each other. Using owl axiom owl:sameAs (for individuals) and owl:equivalentTo (for concepts) we have been able to map AJP-4.10.A triage statuses with the S.M.A.R.T. methodology statuses and more found in crisis management methodologies.

To ensure efficiency of classification, a decision was made to restrain the concept list. This ensures model readability

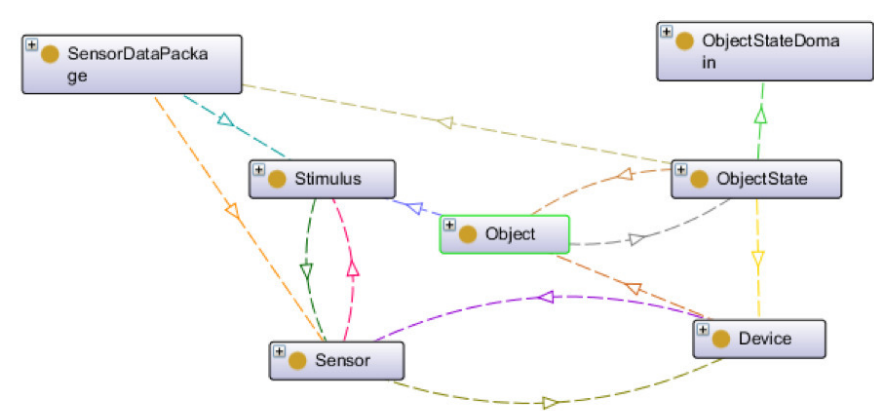

Fig. 3. Combat Triage Ontology core concepts and object properties formin available asociations between instance data

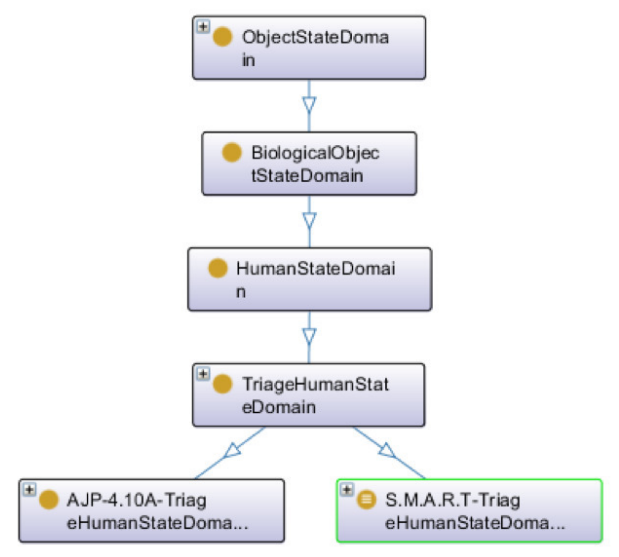

Fig. 4. Combat Triage Ontology core concepts and object properties formin available asociations between instance data

and supports future modularisation. The ontology has been developed using OWL 2.0 RL [10] merging OWL and SWRL language capabilities. Final form of the ontology has been developed using SHOIN(D) Description Logic dialect [11], which demonstrates complexity of ontology definitions, containing over 20 SWRL rules, 50 concepts, 20 object properties and over 20 datatype properties. One of the important characteristics of the ontology is that it contains over 29 defined concepts implementing both value and cardinality restrictions, moreover the model has been modularised consisting of three parts: base terminology, defined concepts terminology and instance base. Such construction supports further extensions of terminology but most of all separates meta model from data instances and prepares the knowledge base to be fed wit data from the real sensor system or simulation software.

\section{Simulation EXPERIMENTS}

\section{A. SenseSim simulator}

To verify presented approach we used SenseSim simulator [12]. It is capable of simulating smart sensors or devices both connected in WSN and IoT. It focuses less on technical aspects of wireless communications, so its communication model is idealistic and does not cover all low level issues [13]. That is 
because SenseSim is designed to simulate sensors network as an autonomous, self organizing system, which is embedded in the environment, where many different phenomena may occur. It focuses on the interaction of smart devices with the external world by the process of perception. One of the key feature of the simulator is that it allows flexibly define sensors attached to the device. This is possible thanks to usage of formal model of perception [14]. It bases on a construct of observers. An observer is a six-tuple:

$$
\langle(X, \chi),(Y, \nu), E, S, \pi, \eta\rangle
$$

which satisfy the following conditions:

1) $(X, \chi)$ and $(Y, \nu)$ are measurable spaces; $E \in \chi$ and $S \in \nu$.

2) Map $\pi: X \rightarrow Y$ is a measurable surjective function with $\pi(E)=S$.

3) Let $(E, \varepsilon)$ and $(S, \varsigma)$ denote the measurable spaces on $E$ and $S$ respectively induced from those of $X$ and $Y$. Then $\eta$ is a statistic kernel on $S \times \varepsilon$ such that, for each point $s \in S, \eta(s)$ is a probability measure supported in $\pi^{-1}\{s\} \cap E$.

When $O$ observes it does not interact with the object of perception itself. Space $X$ is a mathematical construct and is called configuration space. It represents all properties of relevance to $O$. Space $Y$ is a formal representation of premises about events which occur in $X$. Based on those premises the observer can conclude what happen in the external world. Set $E$ is called a distinguished configuration and represents events of interest of an observer. Set $S$ is called distinguished premises and holds the premises about event $E$. Transformation between spaces $X$ and $Y$ is realized by function $\pi$, called perspective. Let us suppose that some point $x \in X$ represents the property of relevance to $O$. Then $O$, in consequence of interaction with the outside world, does not see $x$ but its representation $y=\pi(x)$, where $y \in Y$. If $x$ is in $E$ then $y$ is in $S$. However all that $O$ receives is $y$, not $x$. In other words, the observer must decide whether event $E$ really occurred, basing on premises $S$. Function $\pi$ is surjection, so $O$ does not really know which point $x \in E$ corresponds to given point $y \in S$. That is why with observer's definition comes conclusion kernel $\eta$. It provides, for each point in $S$, the probability distribution supported on $E$. $\eta$ gives the final result of the observer - the probability that for given premises $S$ event $E$ occurred in the real world.

For instance consider an electronic thermometer (which can be one of the sensors used in a triage process). One of the most common are resistance thermometers, for example PT100 [15]. In this case the space $X$ is a temperature of an object in the external (for the observer) ,world". The role of thermometer is to ,guess" as accurately as possible what is its value. It is know, physical fact that resistance of some materials may change according to temperature and that is why resistance thermometers consist of some resistor, for instance platinum. So, what thermometer really knows is the current resistance, which is considered as an element of space $Y$.
Basing on this knowledge thermometer concludes what is the value of temperature in the external world. For example PT100 thermometer has a built in table which maps resistance into temperature. In fact it is an implementation of the conclusion kernel $\eta$.

Another example of observer can be infrared camera. In this case the space $X$ is a three dimensional scene (in the infrared light spectrum). The space $Y$, on the other hand, is two dimensional space which represents the projection of the $3 \mathrm{D}$ scene onto digital image sensor. The $\pi$ function describes how this projection is done. For instance it can be standard perspective projection which angle is determined by the focal length of the sensor's lens. At this point the observer has some premises (space $Y$ ) about the external world (space $X$ ). Let us assume that considered observer is designed to distinguish objects like human thermal image. In this case its distinguished configuration $E$ is this part of the $3 \mathrm{D}$ scene with a human. Accordingly its distinguished premise $S$ is 2D projection of the scene. All the observer knows about the external world is its premise so it uses the conclusion kernel $\eta$ to decide if there is really a human. In this case $\eta$ is more complicated than in previous example and should consists of a pattern recognition methods.

Implementation of the Theory of Perception in SenseSim simulator gives great flexibility in designing sensors which are used in experiments. It allows us to model wide variety of observers, both real and futuristic, which can provide perceptual capabilities for the simulated devices.

SenseSim has quite idealistic model of communication [12], however the network model allows to define heterogenous wireless networks, with fixed or ad-hoc topology as well as different communication interfaces. Network of devices $(D N)$ is modeled as an Bounded Independence Graph [16]:

$$
D N(t)=\left\langle D E V, E^{D N}(t), \Upsilon^{D N}(t)\right\rangle
$$

Where:

- $D E V$ - a set of devices,

- $\Upsilon^{D N}(t)$ - set of independent devices: $\Upsilon^{D N}(t)=$ $\left\{\left\{\operatorname{dev}_{u}, \operatorname{dev}_{v}\right\}: \operatorname{dev}_{u}, \operatorname{dev}_{v} \in D E V ; \operatorname{dev}_{u} \neq \operatorname{dev}_{v}\right\}$

- $E^{D N}(t)$ - a set of edges at time $t$, which is defined as:

$$
\begin{gathered}
E^{D N}(t)=\left\{\left\{\operatorname{dev}_{x}, \operatorname{dev}_{y}\right\}: \operatorname{dev}_{x}, \operatorname{dev}_{y} \in D E V\right. \\
\operatorname{dev}_{x} \in N_{\operatorname{dev}_{y}}^{D N}(t) ; \operatorname{dev}_{y} \in N_{\operatorname{dev}_{x}}^{D N}(t) ; \\
\operatorname{dev}_{x} \neq \operatorname{dev}_{y} ; \\
\left.\left.\left\{\operatorname{dev}_{x}, \operatorname{dev}_{y}\right\} \notin \Upsilon^{D N}(t)\right\}\right\}
\end{gathered}
$$

Where $N_{d e v_{x}}^{D N}$ and $N_{d e v_{y}}^{D N}$ are sets of neighbors of device $d e v_{x}$ and $d e v_{y}$ respectivly.

Neighbor for a device $d e v_{x}$ is other device which fulfills the following conditions:

1) Let $\mathrm{Com}^{\text {dev }_{x}} \subset C O M$ be a set of communication interfaces installed on device $d e v_{x}$ and $\operatorname{Com}^{d e v_{y}} \subset C O M$ be a set of communication interfaces installed on device 


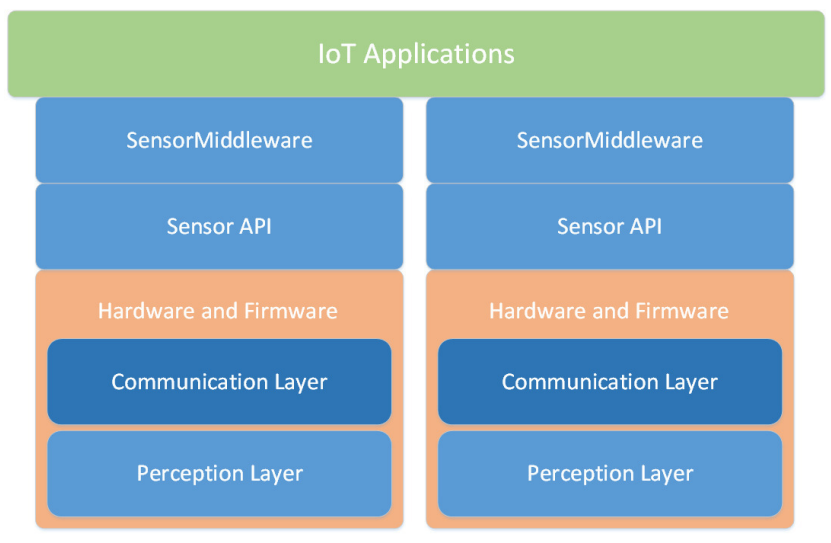

Fig. 5. Sensor's architecture

$$
\begin{aligned}
& d e v_{y} \text { then: }
\end{aligned}
$$

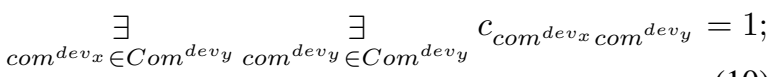

$c_{\text {com }^{\text {dev }} \text { com }^{\text {devy }}}$ is an element of $C M I$ matrix, defined as

$$
C M I=\left[c_{i k}\right]_{|C O M| \times|C O M|}
$$

Where:

- $c_{i k} \in\{0,1\}$ - defines interoperability of $i$-th and $k$ th communication interface. If $c_{i k}=1$ it means that $\mathrm{Com}_{i}$ and $\mathrm{Com}_{k}$ can cooperate with each other. In the opposite case they cannot.

2) Let $l_{d e v_{x}}^{G}(t)=\left\langle l a t_{x}, l_{o n}, e l v_{x}\right\rangle$ be a spatial (geographic) location of device $d e v_{x}$ at time $t$ and $l_{\text {dev }}^{G}(t)=$ $\left\langle l a t_{y}, l_{\text {lon }}, e l v_{y}\right\rangle$ be a spatial (geographic) location of device $d e v_{y}$ at time $t$. Then:

$$
d^{G}\left(l_{\operatorname{dev}_{x}}^{G}(t), l_{\operatorname{dev}_{y}}^{G}(t)\right) \leq \min \left(r(t)_{\operatorname{com}^{\text {dev }_{x}}}, r(t)_{\operatorname{com}^{\text {dev }_{y}}}\right)
$$

Where:

- $r(t)_{\operatorname{com}_{\text {dev }}}$ - radio range of the communication interface of the device $d e v_{x}$ which fulfills first condition.

- $r(t)_{\text {com }^{\text {dev }} y}$ - radio range of the communication interface of the device $d_{e} v_{y}$ which fulfills first condition.

- $d^{G}\left(l_{d e v_{x}}^{G}(t), l_{d e v_{y}}^{G}(t)\right)$ - geographical distance between devices $d e v_{x}$ and $d e v_{y}$

SenseSim has idealistic communication model, because it is focused mainly on cognitive and behavioral aspects of the IoT devices. The simulated devices have multilayer architecture with three main layers (see Figure 5), which is currently standard approach [17] [18]:

- Hardware \& Firmware,

- Sensor API,

- Sensor Middleware.

The first one consists of two other layers: Perception Layer, which is responsible for managing perceptual capabilities, and
Communication Layer, which is responsible for communication issues. The first layer, in the context of simulation, is artificial. On the top of the Hardware, the Sensor API is built. It is an interface which allows to manage the device's hardware from the outside. Our approach to the device's architecture is compliant with IEEE P2413 standard, which specify the Properties layer (in our case the Perception layer), the Information Exchange layer (in our case the Communication Layer) and the Function/Method layer (in our case decomposed into Sensor API and Sensor Middleware layers) [19]. From the point of view of the presented method the most important layer of this architecture is the middleware. To support the triage process we implemented the middleware capable of handling ontologies (especially the triage domain ontology) and infer among them. That makes each device an cognitive agent, which can monitor soldier vital signs using its sensors and understand the measured data. Each of the devices can also receive information need defined in infon logic, interpret it and give as precise as possible response.

Presented method was verified in simulation environment. The main goal of the experiments was to check if the method is suitable for distributed, heterogeneous sensor network system.

\section{B. Simulation results}

Figure 6 shows initial state of the scenario simulated in SenseSim. The network has 15 devices which connect to each other using wireless channel. It is assumed that each device has the same communication capabilities and all messages are sent in peer-to-peer meaner. Topology if the network is not strict and may change in time due to devices movement. Devices can communicate with each other as long as they stay within radio range. In this scenario maximum range is $200 \mathrm{~m}$ and bandwidth of the link is maximum 5 kbps. Devices use flooding routing algorithm for distributing messages. Flooding is not efficient algorithm, it generates a lot of network traffic and may cause redundant messages. On the other hand it gives high probability of message delivery, especially when network topology may change and devices have little or even no knowledge about it.

In this scenario it is assumed that each soldier has one personalised device with connected sensors. For simplicity one device has one of the following sensors attached:

- ECG sensor,

- Pulse oximeter sensor,

- Blood pressure sensor,

- Temperature sensor.

Devices can be interpreted as soldiers' personal computers like in future soldier systems i.e.: FIST [20], IdZ [21], TYTAN [22].

During scenario different information needs were sent into the network form device number 10, which can be considered as a team leader. Figure 7 shows example of information need processing. Device 10 sends at time 19,9 into the network question about the state of the Soldier_3:

$$
\ll \text { ?: ObjectState, Soldier_3, } 1 \gg
$$




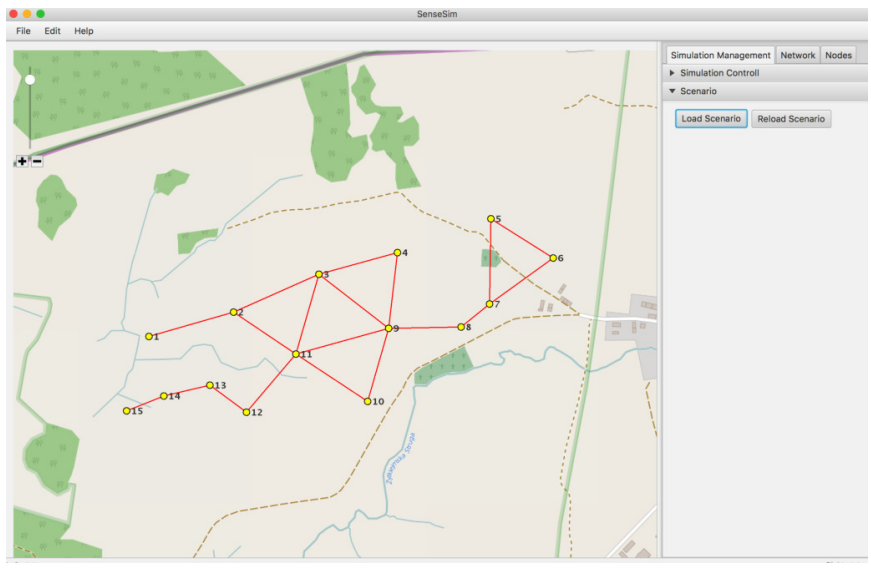

Fig. 6. Simulated network in SenseSim

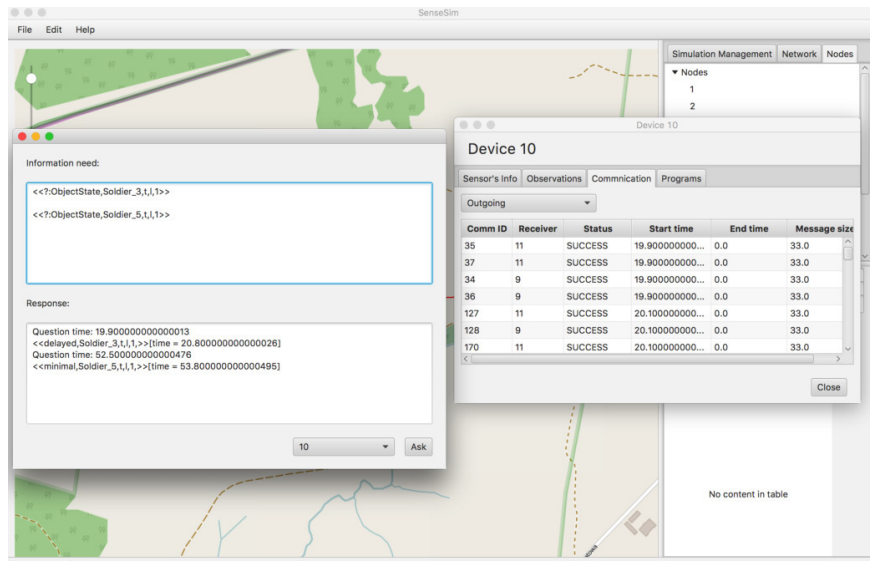

Fig. 7. Example of the question about the state of one soldier

Information need is distributed between devices using flooding algorithm. The role of each middleware is to interpret the need and check if the device can answer it. In this case only the middleware of the device number 3 can fulfil the need (because this device monitors Soldier_3). Basing on ontology reasoning it comes to conclusion that the Soldier_3 is in state delayed. After that device 3 sends response for the need:

$$
\ll \text { delayed,Soldier_3, } 1 \gg \text {. }
$$

It is received at time 20,8 by the asking device 10 .

Figure 8 shows example of different need processing. In this case the network was asked to define which soldiers are in state minimal treatment. Information need was sent at time 34,1 and had the following form:

$$
\ll \text { minimal : ObjectState, } ? h, 1 \gg
$$

This question requires action from every device in the network, because each have to verify the state of monitored soldier and decide if he is in state minimal treatment or not. That is why fulfilling such need is a more complicated process than shown in earlier example. Response for the need circles

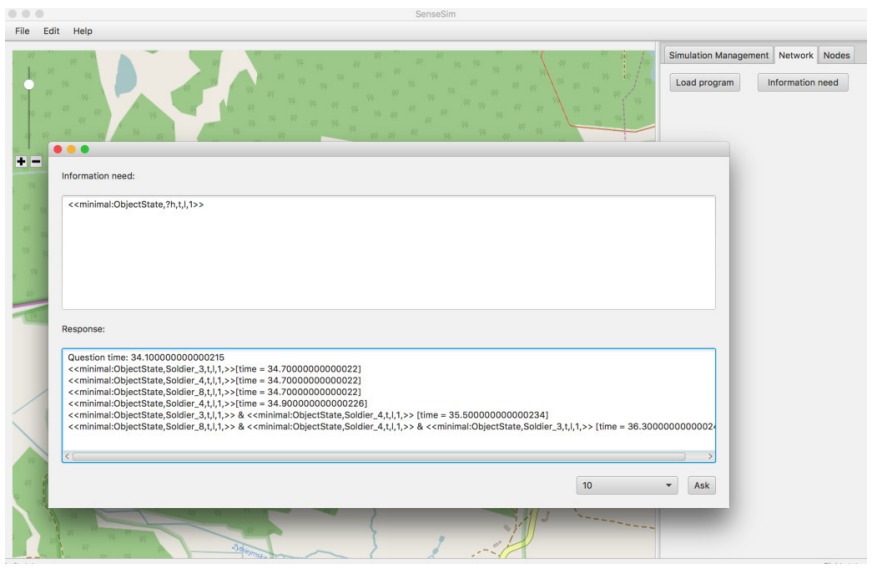

Fig. 8. Example of question about soldiers who are in state minimal

in the network and each device adds a part to it. Because of the flooding algorithm, the asking device also receives parts of the answer and at time 36,3 receives full answer:

$$
\begin{aligned}
& \ll \text { minimal, Soldier_8, } 1 \gg \\
\& & \ll \text { minimal, Soldier_4, } 1 \gg \\
\& & \ll \text { minimal, Soldier_3, } 1 \gg
\end{aligned}
$$

In every simulated scenario devices were able to answer the information need basing on its own knowledge about monitored objects (soldiers).

\section{CONCLUSION}

In this paper a novel approach to supporting combat triage was presented. Currently developed solutions focus on supporting triage by providing ontology based expert systems [23] [24] or by providing dedicated triage sensors [25] [26]. The first approach gives first responder great support, but still requires a lot of resources and attention to monitor wounded continuously. The second approach is more automatic, but also less flexible. It requires that every wounded has the same sensor for health monitoring. If they are not available, manual work is needed. Our approach is more flexible. It does not need any additional infrastructure than sensors network. Also we do not close our method to particular sensors. In fact any sensor adequate for triage process can be used by our approach. Moreover we do not limit sensors only to wearable devices. Soldier's state may be evaluated also by nearby sensors, for instance those installed in vehicle. From the perspective of end user it is transparent.

Thanks to ontology reasoning and presented information framework, an expert knowledge is not required from first responders to carry out the triage process properly. Some vital signs registered by sensors, like ECG, may be hard to interpret without medical background, but there are methods for automatic processing and extracting valuable information. Moreover using IoT devices for triage, makes this process to become continuous with minimal manual effort. 


\section{REFERENCES}

[1] P. P. Jayaraman, K. Gunasekera, F. Burstein, P. D. Haghighi, H. S Soetikno, and A. Zaslavsky, "An ontology-based framework for realtime collection and visualization of mobile field triage data in mass gatherings," in System Sciences (HICSS), 2013 46th Hawaii International Conference on, Jan 2013, pp. 146-155.

[2] N. Gilboy, P. Tanabe, D. Travers, and A. Rosenau, Emergency Severity Index (ESI) A Triage Tool for Emergency Department Care. AHRQ Publication No. 12-0014, November 2011.

[3] D. Meenach, "How civilian and combat triage differ," Apr 2015. [Online]. Available: http://www.ems1.com/columnists/dean-meenach/ articles/2147174-how-civilian-and-combat-triage-differ/

[4] “Tactical combat casualty care." [Online]. Available: http://www.naemt org/education/tccc/tccc.aspx

[5] AJP-4.10(A) - Allied Joint Medical Support Doctrine. Nato Standardization Agency (NSA), March 2006.

[6] K. J. Devlin, Logic and information. Cambridge University Press, 1991.

[7] M. Dyk, A. Najgebauer, and D. Pierzchala, Augmented perception using Internet of Things. Oficyna Wydawnicza Politechniki Wroclawskiej, 2014, pp. 109-118.

[8] F. Baader, D. Calvanese, D. L. McGuinness, D. Nardi, and P. F. PatelSchneider, "The Description Logic Handbook: Theory, Implementation, and Applications," Description Logic Handbook, p. 622, 2003. [Online]. Available: http://portal.acm.org/citation.cfm?id=1215128

[9] B. Parsia and E. Sirin, "Pellet : An OWL DL Reasoner," Artificial Intelligence, pp. 1 - 2, 2000.

[10] D. Reynolds, "OWL 2 RL in RIF (Second Edition)," W3C Working Group Note, no. February, pp. 1 - 128, 2013. [Online]. Available: http://www.w3.org/TR/2013/NOTE-rif-owl-rl-20130205/

[11] I. Horrocks and U. Sattler, "Ontology reasoning in the shoq(d) description logic," in Proceedings of the 17th International Join Conference on Artificial Intelligence - Volume 1, ser. IJCAI'01. San Francisco, CA, USA: Morgan Kaufmann Publishers Inc., 2001, pp. 199 - 204. [Online]. Available: http://dl.acm.org/citation.cfm?id=1642090. 1642117

[12] M. Dyk, D. Pierzchala, and A. Najgebauer, SenseSim: An Agent-Based and Discrete Event Simulator for Wireless Sensor Networks and the Internet of Things, 2015, pp. 345-350.

[13] M. Dyk, A. Najgebauer, and D. Pierzchala, Agent-Based M\&S of Smart Sensors for Knowledge Acquisition Inside the Internet of Things and Sensor Networks. Springer International Publishing, 2015, pp. 224 236
[14] B. Bennet, D. Homan, and C. Prakash, Observer Mechanics. A Formal Theory of Perception. Academic Press, 1989.

[15] “Omega engineering." [Online]. Available: http://www.omega.co.uk/ prodinfo/rtd.html

[16] S. Schmid and R. Wattenhofer, "Modeling sensor networks," in Algorithms and Protocols for Wireless Sensor Networks, A. Boukerche, Ed. Wiley-IEEE Press, 2008, pp. 77-105.

[17] D. Bandyopadhyay and J. Sen, "Internet of things: Applications and challenges in technology and standardization," Wireless Personal Communications, vol. 58, no. 1, pp. 49-69, 2011.

[18] C. Yuqiang, G. Jianlan, and H. Xuanzi, "The research of internet of things' supporting technologies which face the logistics industry," in Computational Intelligence and Security (CIS), 2010 International Conference on, Dec 2010, pp. 659-663.

[19] O. Logvinov. (2015) Standard for an architectural framework for the internet of things. [Online]. Available: http://grouper.ieee.org/groups/ 2413/Intro-to-IEEE-P2413.pdf/

[20] "Fist - future infantry soldier technology." [Online]. Available: http://www.army-technology.com/projects/fist/

[21] "Idz (infanterist der zukunft) future soldier system." [Online]. Available: http://www.army-technology.com/projects/idz/

[22] N. P. V. 11 January, 2012 Âu Industry Profiles, "Uhlan 21: The polish future soldier project.” [Online]. Available: http://www.sadefensejournal. $\mathrm{com} / \mathrm{wp} / \mathrm{p}=912$

[23] S. H. Regli, "C4isr-med battlefield medical demonstrations and experiments," ILockheed Martin Advanced Technology Laboratories, 2012.

[24] T. Gao, C. Pesto, L. Selavo, Y. Chen, J. Ko, J. Lim, A. Terzis, A. Watt, J. Jeng, B.-R. Chen, and et al., "Wireless medical sensor networks in emergency response: Implementation and pilot results," 2008 IEEE Conference on Technologies for Homeland Security, 2008.

[25] P. Shaltis and A. Reisner, "Rapidly deployable sensor design for enhanced noninvasive vital sign monitoring," Jul. 1 2010, uS Patent App. 12/604,043. [Online]. Available: http://www.google.ch/patents/ US20100168531

[26] "U.s. military develops blood-loss detection device that predict shock." [Online]. Available: http://www.meddeviceonline.com/doc/ u-s-military-develops-finger-worn-device-that-detects-blood-loss-0001 